\section{Intravenous infusions and one-way valves}

To the Editor:

I would like to report a situation recently encountered during anaesthetic infusion. A 60 -yr-old man presented for elective repair of a tom right rotator cuff. General anaesthesia was induced with propofol administered through an intravenous line inserted into a left forearm vein. Anaesthesia was maintained with an infusion of propofol, using a Bard Mini-infuser system, piggybacked into an injection port of a Baxter Continu-flo solution set. With each inflation of the automated blood pressure cuff, on the left upper arm, it was apparent that propofol was refluxing up past the check valve and into the drip chamber of the solution set. The check valve was examined, the disc appeared intact and no air bubbles were seen. Tapping the valve had no effect. The infusion rate was increased slightly and this was sufficient to keep retrograde flow below the level of the drip chamber during cuff inflation. After completion of the surgery the patient was awakened, taken to the recovery room and, before he was sent to the ward, the solution set was changed. The manufacturer (Baxter Corporation) was notified of the incident.

The one-way valve consists of a silicone rubber disc suspended on supports between the upper and lower halves of the valve. Pressure from above causes the outer edge of the disc to bend down and open the passages to downstream flow (Figure 1). Conversely, pressure from below will lift the disc against the upper portion of the valve and prevent flow back into the drip chamber (Figure 2). Because of the close tolerances throughout the assembly, it is possible that an outer edge of the valve could touch a wall of the valve housing and fail to lift. It is also possible for an air bubble to lodge in the fluid path and prevent the valve from lifting, making it essential to purge the assembly of air during the initial setup. Baxter Technical Services recommends that if back flow is observed, a quick knock on the valve housing will usually be enough to free the disc and restore proper valve function (personal communication).

It is likely that, had a clear solution been infused, the diagnosis of the valve failure would have been delayed or missed. This could have resulted in backward flow of the infusion agent into the bag of intravenous solution and, after the infusion pump was turned off, the infusion agent would continue to be delivered to the patient. The use of intravenous infusions is becoming increasingly common in anesthesia and anaesthetists should be aware of these technical problems.

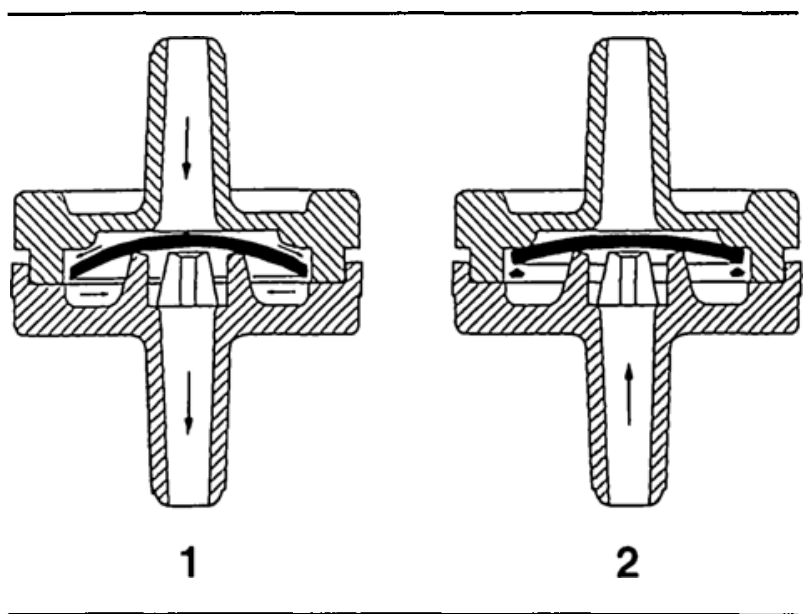

FIGURES I and 2.

Edward Crosby BSC MD FRCPC

Department of Anesthesia

Ottawa General Hospital

501 Smyth

Ottawa, Ontario

KIH 8L6

\section{REPLY}

The Continu-Flo sets are designed with a check valve to prevent back flow of fluid from a secondary intravenous application into the primary container. Millions are sold annually in Canada and the USA. In order to ensure strict quality control, each set is inspected at every stage of the manufacturing process. The quality process ensures that Baxter's complaint frequency is very low, averaging one complaint for every 1.5 million sets sold in Canada.

Instructions for priming are printed on the packaging of every iv administration set that Baxter manufactures. Solution "Back Flow" or leakage of solution through the check valve's floating disk can occur as stated by Dr. Crosby. Our experience has found that if this occurs, tapping of the valve with a rigid instrument such as a haemostat or a pair of scissors, will dislodge any air bubbles or free the disk and stop the back flow. If back flow does occur, it will be evident the first time a back pressure is applied to the valve. This will not occur intermittently, it will be immediately visible to the anaesthetist. If back flow occurs there will be a rise in the fluid level and flooding of the primary set drip chamber and the anaesthetist can take appropriate action.

If there is any doubt about the integrity of any component of an iv administration set, the set should be replaced immediately. $A$ refund or credit will be issued to the hospital by Baxter Corporation for a manufacturer's defect in the product.

If questions should arise regarding the use of Baxter iv administration sets with another manufacturer's product or for a special procedure, the local Baxfer representative would be able to detail the proper protocol to ensure a safe and 